\title{
Ecological role of homeless dogs in anthropogenic territories
}

\author{
I.I. Rakhimov ${ }^{1}$, E.Sh. Shamsuvaleeva ${ }^{2}$, A.V. Arinina ${ }^{l}$ \\ ${ }^{1}$ Kazan (Volga Region) Federal University, Kazan, Russia \\ ${ }^{2}$ Povolzhskaya State Academy of Physical Culture, Sports and Tourism, Kazan, Russia
}

\begin{abstract}
The article presents materials of long-term observations for homeless dogs in the territory of the Republic of Tatarstan. Dogs that have passed the path of their domestication are currently showing the opposite process - feralization. According to unofficial data, there are tens of thousands of stray animals in Kazan alone. As the dominant land-based predators, and not only in cities, but also in the suburbs of settlements, they pose a threat to biodiversity in various ecosystems. Stray dogs are indicated as a limiting factor for the large jerboa and speckled ground squirrel included in the Red Book of the Republic of Tatarstan.
\end{abstract}

\section{Introduction}

Among the most pressing modern problems is the problem of finding out the possibilities and limits of stability of multicomponent ecosystems. The very fact of the attainability of sustainable development depends on the ability to predict the behavior of ecosystems. The development of methods of rational nature management is based on the identification of natural mechanisms for maintaining biological diversity, in particular, the study of animals activities in order to predict the consequences of their activities and the search for ways to influence this activity.

In the domestic scientific literature animals that transform their habitat and affect the surrounding biotic and abiotic objects are usually called environment-transforming ones, and their activity is called environment-transforming. A similar term - keystonespecies - is common abroad and is understood as a living organism that has special and disproportionately large effect on other organisms and ecosystem processes. Its role is determined not by the amount of biomass, but by the volume of "biogeocenotic work" performed [1].

\section{Results and Discussion}

Nowadays the presence of homeless animals, whose biocenotic role is clearly manifested, is practically observed everywhere, for which cause their environmental-transforming role requires active research. The result of stray dogs habitation is the transformation of the environment through a change in the species composition of ecosystems, that significantly reduces the adaptive potential of ecosystems to their self-organization. 
The aim of the study is a natural-scientific analysis of the ecological role of stray dogs in anthropogenic territories in the process of their reverse feralization. The joint development of anthropogenic biotopes and the inevitability of contacts determine the multiple ecological ties between domestic and wild animals, which go beyond the classical framework: they can be weakened, strengthened or distorted. Dogs have gone through a unique path of their domestication, but now we have to observe the process of reverse domestication - the feralization of stray dogs.

In the context of Tatarstan ecosystems conditions there should be a wolf or a bear at the top of the food pyramid. According to the data of the Tatarstan State Committee for Bioresources there have been performed accountings, that showed the presence of only 17.7 thousand predatory animals. The most widespread predators in the territory of the republic are the fox and the badger. The number of some wild animals does not exceed several dozen, for example, lynx and corsac. There are about two dozens of wolves in the republic. Bears can be found in the republic, but their exact number has not been established. The dynamics of the wolf population, which has significantly decreased over the past twenty years, is of greatest interest (Fig. 1).

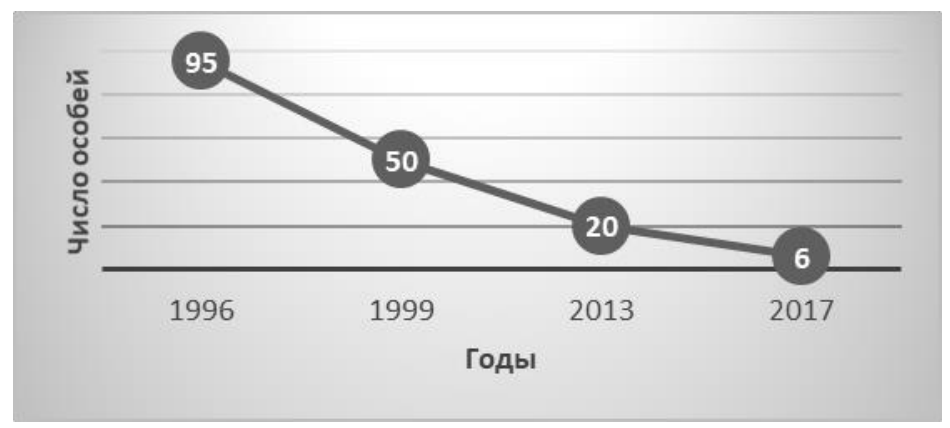

Fig. 1. Dynamics of the wolf population in the Republic of Tatarstan.

It is obvious that the number of stray dogs is several orders of magnitude greater than the number of wolves, which requires reasonable measures to regulate their numbers in order to preserve the species diversity of wild animals living in the republic. According to unofficial data, there are tens of thousands of stray animals in Kazan alone.

Studies in various regions have also shown that the real displacement of feral and stray dogs from the territory occurs in case of wolf habitation in this area. According to the Chronicle of Nature in the territory of the Volga-Kama Nature Reserve the presence of a wolf is the most significant factor regulating the number of stray dogs.

Homeless dogs have been studied as the dominant predators, which pose a threat to biodiversity in many regions, including Moscow, St. Petersburg, Samara, Omsk, Novosibirsk, etc. Thus, in the Red Book of the Republic of Tatarstan stray dogs are officially indicated as a limiting factor for two species of mammals: the great jerboa and the speckled ground squirrel.

Birds nesting on the ground and in man-made structures are potentially the most accessible prey for stray animals. Long-term observations show a significant decrease in the number of ground-nesting birds or their complete absence in the territories of Kazan forest and parkland zones accessible to dogs and cats. Wood grouse, black grouse, hazel grouse, woodcock, snipe, nightjar have become rare. 92 species of Tatarstan birds have their nests in the dogs' reach zone. Particular emphasis needs to be placed on 21 species of birds already included in the Red Book of the Republic. Dogs hunt mouse-like rodents, marmots, badgers, young wild boars, they can drive roe deer and elk. The pursuit of ungulates by packs of stray dogs has been noted in different regions of the republic. Feral dogs are enemies of beavers and minks. They manage to dig a ground squirrel, driven into protective 
or wintering burrows. They are able to eliminate the ground squirrel colony over the summer. Almost $30 \%$ of deaths of raccoon dogs are due to encounters with feral dogs. They hunt hares by "driving" them. They even chase hedgehogs, forcing them to change their habitat. In the forest-park belt of the city amphibians and reptiles suffer from stray dogs: newts, common spadefoot, fire-bellied toad, green and common toads, grass, moor, lake, edible and pool frogs, sand and viviparous lizards, anguine lizard, grass snake.

In the anthropogenic landscape the relationships between a fox and stray dogs show itself in competition not only for food, but also for territory. Due to the similarity of hunting objects, using of garbage at dump sites and cattle burial grounds for food, the settlement at the same type of shelters located in the same biotopic conditions, the competition between dogs and foxes intensifies, moreover foxes visit such places only after the dogs leave. Many authors point out that stray dogs take refuge in burrows of foxes, badgers and raccoon dogs. Feral dogs dig foxes out of their burrows, occupy their burrows.

Winter accounting on tracks in the vicinity of Kazan showed that the more tracks of dogs, the fewer tracks of both a hare and a fox; with a decrease in the number of dog tracks, the number of hares' and foxes' tracks increases. The very fact of the confirmed predation of stray dogs in relation to the fox is important. The Chronicle of Nature of the Volga-Kama State Natural Biosphere Reserve notes that the constant presence of feral dogs leads to the displacement of foxes from the territory of the reserve [2]. The number of grouse birds and hares negatively correlates with the number of dogs.

In the Moscow region facts of attacks by packs of stray dogs have been established against elks, sika deer, roe deer, young wild boars, ermines, ferrets, badgers, hares, birch mice, squirrels, hedgehogs, and ground-nesting birds [3]. In winter, with a snow cover of 50 $\mathrm{cm}$, roe deer are easy prey for wild dogs wandering in hunting grounds. For example, in the Samara region, 2019 became a record year for the number of dead roe deer, when the death toll numbered into dozens of animals. [4].

The exterminating nature of hunting of stray and homeless dog packs causes irreparable harm to the hunting industry. In the absence of selectivity in hunting, the destruction or displacement of all who can be overcome, occurs. It is also worth noting that there are 48 types of helminths common for dogs and other warm-blooded vertebrates, as well as common infections such as brucellosis, listeriosis, piroplasmidosis, rabies, leptospirosis, tuberculosis.

These facts demanded urgent measures on reduction in the number and on reproduction rate decreasing of feral dogs. The solution to this problem is not so straightforward, since there are certain social aspects associated with the reduction in the number of dogs. Such methods as trapping, sterilization and return to the medium were initially thought to be effective, for example, from the late 1990s in Moscow. However, at present, these techniques are recognized as ineffective, which is confirmed by numerous studies $[5,6,7$, $8,9,10]$.

\section{Conclusions}

Feral, stray and domestic dogs are now found in almost all regions of the Earth and outnumber all other canines. Among the factors that led to the possibility of reverse feralization of dogs is a decrease in the number of wild predators, in particular the wolf. The aggregated spatial pattern and density of stray dogs subpopulations contribute to the spread of infectious and invasive diseases in the subpopulation itself, in populations of wild animals, as well as among humans. Without serious scientifically grounded support for the problem of stray animals, stray dogs become a threat to the number of natural populations of prey species and, as a consequence, to the stability of natural and anthropogenic ecosystems. 


\section{References}

1. O.A. Bukhareva, Ecological functions of burrowing systems of small mammals in different natural zones of the European territory of Russia: Dis...Cand. Sc. Biology, (2014)

2. I. Brickner, The impact of domestic dogs (Canisfamiliaris) on wildlife welfare and conservation: a literature review. With a situation summary from Israel. (Tel Aviv University, 2003)

3. A.O. Vereshchagin, A.D.Poiarkov, P.V. Rusov and others, Census of homeless and ownerless animals (dogs) in the territory of Moscow Domestic dog research problems: Meeting materials, 95-114 (2006).

4. For hunting providers about strengthening the fight against wild dogs: [Electronic resource] (Hunting and Fishing Department of the Samara region)

URL: https://dor.samregion.ru/2020/01/13/ohotpolzovatelyam-ob-usilenii-borby-sdikimi-sobakami/

5. D.V.Zaloznykh, O.I. Ponomarenko, Veterinary pathology, 2 , 19-23 (2006).

6. G.B. Poyaganov, Veterinary pathology, 2, 7-12 (2006).

7. A.I. Rakhmanov, Veterinary pathology, 1, 136 (2002).

8. V.A. Rybalko, Review of world experience in solving the problem of homeless animals, Veterinary pathology, 2(17) (2006).

9. E.Sh.Shamsuvaleeva, A.V.Arinina, N.V. Salakhov, Fundamental studies, 9(1), 91-95 (2015).

10. E.Sh.Shamsuvaleeva, I.I. Rakhimov, Peculiarities of stray dogs ecology in conditions of the city of Kazan and its suburbs ("Novoe znanie", Kazan, 2013). 